\title{
On grounded L-graphs and their relatives
}

\author{
Vít Jelínek* \\ Computer Science Institute, Faculty of Mathematics and Physics, \\ Charles University, Prague, Czechia \\ jelinek@iuuk.mff.cuni.cz \\ Martin Töpfer ${ }^{\dagger}$ \\ Institute of Science and Technology, Klosterneuburg, Austria \\ mtopfer@gmail.com
}

Submitted: Aug 16, 2018; Accepted: Jul 1, 2019; Published: Jul 19, 2019

(C) The authors. Released under the CC BY license (International 4.0).

\begin{abstract}
We consider the graph classes Grounded-L and Grounded- $\{L\rfloor$,$\} correspond-$ ing to graphs that admit an intersection representation by L-shaped curves (or Lshaped and $\lrcorner$-shaped curves, respectively), where additionally the topmost points of each curve are assumed to belong to a common horizontal line. We prove that GROUNDED-L graphs admit an equivalent characterisation in terms of vertex ordering with forbidden patterns.

We also compare these classes to related intersection classes, such as the grounded segment graphs, the monotone L-graphs (a.k.a. max point-tolerance graphs), or the outer-1-string graphs. We give constructions showing that these classes are all distinct and satisfy only trivial or previously known inclusions.
\end{abstract}

Mathematics Subject Classifications: 05C62, 05C10, 05C75

\section{Introduction}

An intersection representation of a graph $G=(V, E)$ is a map that assigns to every vertex $x \in V$ a set $s_{x}$ in such a way that two vertices $x$ and $y$ are adjacent if and only if the two corresponding sets $s_{x}$ and $s_{y}$ intersect. The graph $G$ is then the intersection graph of

*Supported by the project 18-19158S of the Czech Science Foundation and by project Neuron Impuls of the Neuron Fund for Support of Science.

†This project has received funding from the European Union's Horizon 2020 research and innovation programme under the Marie Skłodowska-Curie Grant Agreement No. 665385. 
the set system $\left\{s_{x} ; x \in V\right\}$. Many natural graph classes can be defined as intersection graphs of sets of a special type.

One of the most general classes of this type is the class of string graphs, denoted STRING. A string graph is an intersection graph of strings, which are bounded continuous curves in the plane. All the graph classes we consider in this paper are subclasses of the class of string graphs.

A natural way of restricting a string representation is to impose geometric restrictions on the strings we consider. This leads, for instance, to segment graphs, which are intersection graphs of straight line segments, or to L-graphs, which are intersection graphs of L-shapes, where an L-shape is a union of a vertical segment and a horizontal segment, in which the bottom endpoint of the vertical segment coincides with the left endpoint of the horizontal one. Apart from L-shapes, we shall also consider $\lrcorner$-shapes, which are obtained by reflecting an $L$-shape along a vertical axis.

Apart from restricting the geometry of the strings, one may also restrict a string representation by imposing conditions on the placement of their endpoints. Following the terminology of Cardinal et al. [4], we will say that a representation is grounded if all the strings have one endpoint on a common line (called grounding line) and the remaining points of the strings are confined to a single open halfplane with respect to the grounding line. We will usually assume that the grounding line is the $x$-axis, and the strings extend below the line. The endpoint belonging to the grounding line is the anchor of the string.

Similarly, a string representation is an outer representation, if all the strings are confined to a disk, and each string has one endpoint on the boundary of the disk. The endpoint on the boundary is again called the anchor of the string. One may easily see that a graph admits a grounded string representation if and only if it admits an outerstring representation. Such graphs are known as outer-string graphs, and we denote their class by OUTER-STRING.

Our first main result, Theorem 1 in Section 2, is a characterisation of the class of grounded L-graphs by vertex orderings avoiding a pair of forbidden patterns. Our next main result, presented in Section 3, is a collection of constructions providing separations between the classes in Figure 1, showing that there are no nontrivial previously unknown inclusions among them.

Let us now formally introduce the graph classes we are interested in, and briefly review some relevant previously known results.

1-string graphs are the graphs that admit a string representation in which any two distinct strings intersect at most once. The class of 1-string graphs is denoted 1-STRING.

Outer-1-string graphs (denoted OUTER-1-STRING) are the graphs that have a string intersection representation which is simultaneously a 1-string representation and an outerstring representation. Note that not every graph from 1-STRING $\cap$ OUTER-STRING is necessarily in OUTER-1-STRING, as we shall see in Section 3.

$\mathrm{L}$-graphs $(\mathrm{L})$ are the intersection graphs of L-shapes. This type of representation has received significant amount of interest lately. A notable recent result is a theorem of Gonçalves, Isenmann and Pennarun [9] showing that every planar graph is an L-graph. Since it is known that L-graphs are a subclass of segment graphs [12], this result strength- 


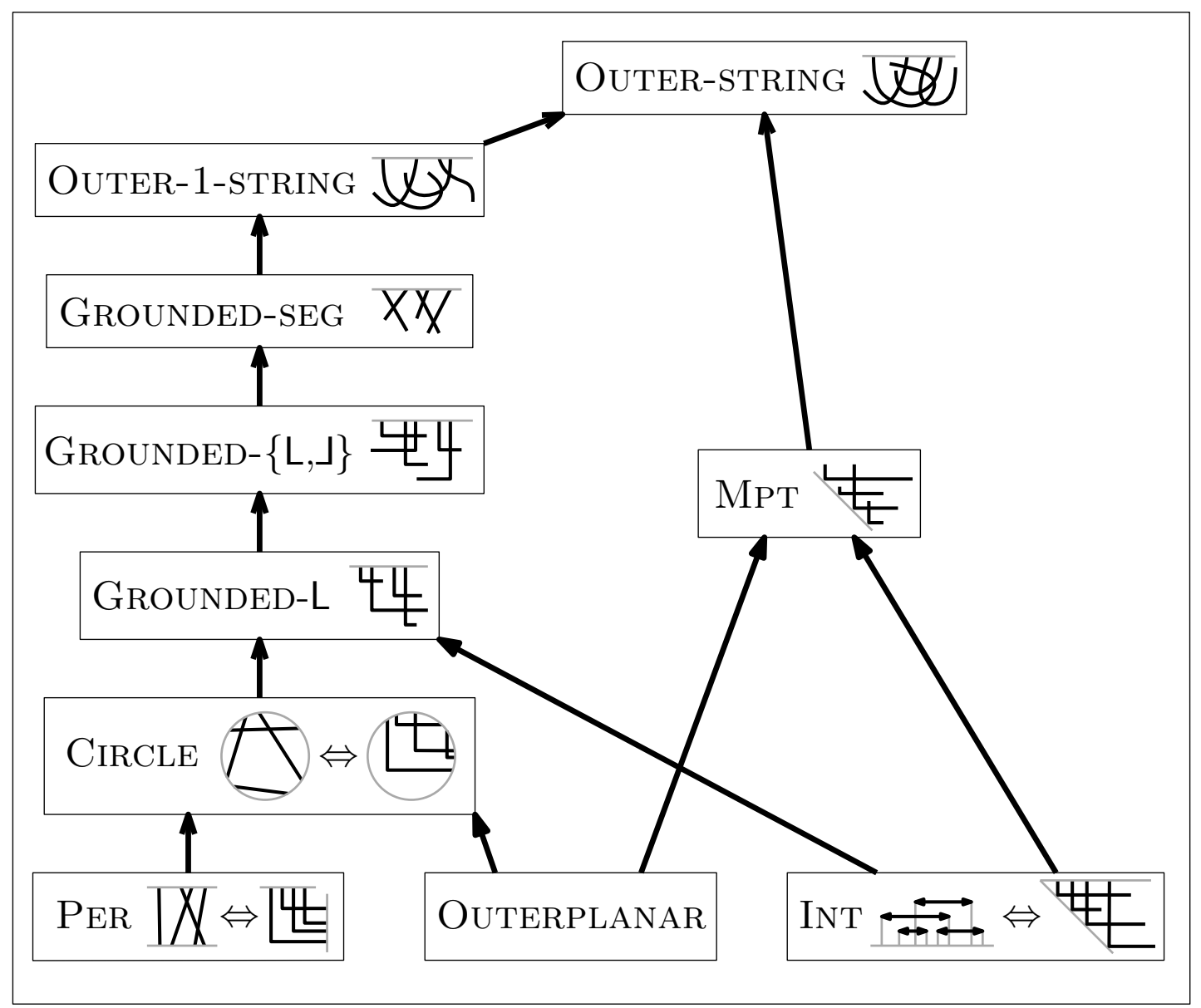

Figure 1: Graph classes considered in this paper. Arrows indicate inclusions. We will see in Section 3 that there are no other inclusions among these classes apart from those implied by the depicted arrows. In particular, the classes are all distinct.

ens an earlier result of Chalopin and Gonçalves [6] showing that all planar graphs are segment graphs.

Max point-tolerance graphs (MPT), also known as monotone L-graphs, are the graphs with an L-representation in which all the bends of the L-shapes belong to a common downward-sloping line. This class was independently introduced by Soto and Thraves Caro [15], by Catanzaro et al. [5] and by Ahmed et al. [1]. Apart from the above intersection representation by L-shapes, it admits several other equivalent characterisations. Notably, MPT graphs can be characterised as graphs that admit a vertex ordering that avoids a certain forbidden pattern $[1,5,15]$. This graph class is known to be a superclass of several important graph classes, such as outerplanar graphs and interval graphs, among others $[1,5,15]$.

Grounded segment graphs (GROUNDED-SEG) are the intersection graphs admitting a grounded segment representation. Cardinal et al. [4] proved that these are also precisely the intersection graphs of downward rays in the plane. Note that any grounded segment 
graph also admits an outer-segment representation, but the converse does not hold, as shown by Cardinal et al. [4]. They also showed that outer-segment graphs form a proper subclass of the class of outer-1-string graphs. This strengthens an earlier result of Cabello and Jejčič [3], who showed that outer-segment graphs are a proper subclass of the class of outer-string graphs.

Grounded L-graphs (GROUNDED-L) are the intersection graphs of grounded L-shapes, that is, L-shapes with top endpoint on the $x$-axis. This class of graphs was first considered by McGuinness [11], who represented them as intersection graphs of upward-infinite L-shapes. These graphs can also equivalently be represented as intersection graphs of L-shapes inside a disk, with the top endpoint of each L-shape anchored to the boundary of the disk. McGuinness showed that this class is $\chi$-bounded, that is, these graphs have chromatic number bounded from above by a function of their clique number. The $\chi$-boundedness result was later generalized to all outer-string graphs by Rok and Walczak [14].

Grounded $\{\mathrm{L}\rfloor$,$\} -graphs (GROUNDED- \{\mathrm{L}\lrcorner$,$\} ) are analogous to grounded L-graphs, but$ their representation may use both $L$-shapes and $\lrcorner$-shapes. An argument of Middendorf and Pfeiffer [12] shows that Grounded- $\{\mathrm{L}\rfloor$,$\} is a subclass of Grounded-SEG.$

Circle graphs (CIRCLE) are the intersection graphs of chords inside a circle, or equivalently, the intersection graphs of L-shapes drawn inside a circle, so that both endpoints of each L-shape touch the circle. Circle graphs include all outerplanar graphs [16].

Interval graphs (INT) are the intersection graphs of intervals on the real line. Equivalently, we may easily observe that these are exactly the graphs with an intersection representation which is simultaneously an MPT-representation and a GROUNDED-L-representation. But note that not every graph from the intersection of MPT and GROUNDED-L is an interval graph, as witnessed, for example, by any cycle $C_{n}$ of length $n \geqslant 4$.

Permutation graphs (PER) are the intersection graphs of segments between a pair of parallel lines, with each segment having one endpoint on each of the two lines. Equivalently, we may observe that these are exactly the graphs admitting an L-representation in which the top endpoints of all the L-shapes are on a common horizontal line and the right endpoints are on a common vertical line.

We will always assume implicitly that the intersection representations we deal with satisfy certain non-degeneracy assumptions. In particular, we will assume that the strings have no self-intersections, that any two strings intersect in at most finitely many points (except for interval representations), and that any intersection of two strings is a proper crossing. In particular, an endpoint of a string does not belong to another string. Moreover, we will assume that every segment in a segment representation is non-degenerate, that is, it has distinct endpoints. This also applies to horizontal and vertical segments forming an $\mathrm{L}$-shape or $\lrcorner$-shape. These assumptions imply, in particular, that in any $\{\mathrm{L}\lrcorner$,$\} -$ representation, each intersection is realized as a crossing of a horizontal segment with a vertical one.

Note that in any grounded representation with a horizontal grounding line, the left-toright ordering of the anchors on the grounding line defines a linear order on the vertex set of the represented graph. We say that this linear order is induced by the representation. 


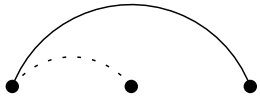

interval graphs

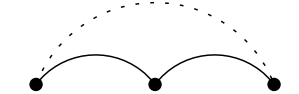

comparability graphs

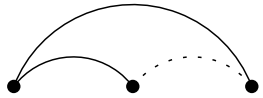

chordal graphs

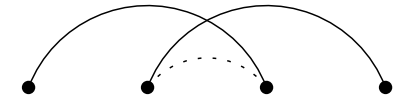

max point-tolerance graphs

Figure 2: Forbidden order patterns for various graph classes [2, 5, 7]. The solid arcs denote compulsory edges and the dotted arcs are forbidden edges.
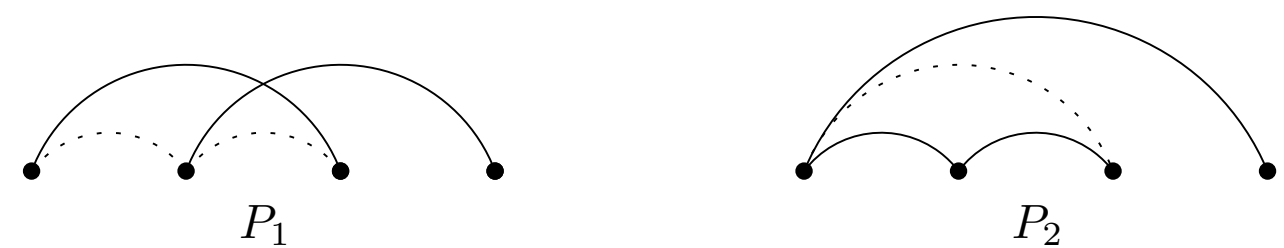

Figure 3: The two forbidden ordering patterns for the class GROUnDED-L.

Similarly, for an MPT representation, we can define the induced order by following the top-left to bottom-right order of the bends along their common supporting line. Induced vertex orders play an important role both in characterising graphs in a given class and in separating different classes.

\section{Vertex orders with forbidden patterns}

Our main result is a characterisation of grounded L-graphs as graphs that admit vertex orderings avoiding a pair of four-vertex patterns. Let us begin by formalising the key notions.

An ordered graph is a pair $(G,<)$, where $G=(V, E)$ is a graph and $<$ is a linear order on $V$. A pattern of order $k$ is a triple $P=(W, C, F)$ where $W$ is the set $\{1,2, \ldots, k\}$ while $C$ and $F$ are two disjoint subsets of $\left(\begin{array}{c}W \\ 2\end{array}\right)$. The set $W$ is the vertex set of the pattern $P$, $C$ is the set of compulsory edges of $P$, and $F$ is the set of forbidden edges.

For an ordered graph $(G,<)$ with $G=(V, E)$, we say that $(G,<)$ contains a pattern $P=(W, C, F)$ of order $k$ if $G$ contains $k$ distinct vertices $x_{1}<x_{2}<\cdots<x_{k}$ such that for every $\{i, j\} \in C$ the vertices $x_{i}$ and $x_{j}$ are adjacent in $G$, while for every $\{i, j\} \in F$, $x_{i}$ and $x_{j}$ are non-adjacent in $G$. If $(G,<)$ does not contain $P$, we say that it avoids $P$. For simplicity, we will often write an edge $\{i, j\}$ as $i j$.

Many important graph classes can be characterised in terms of vertex orderings with forbidden patterns, that is, for a class $\mathcal{C}$ there is a pattern $P_{\mathcal{C}}$ such that a graph $G=(V, E)$ is in $\mathcal{C}$ if and only if it admits a linear order $<$ such that $(G,<)$ avoids $P_{\mathcal{C}}$; see Figure 2 for examples of classes with their forbidden patterns. The forbidden pattern characterisation of MPT was found independently by at least three groups of authors $[1,5,15]$.

As our first main result, we show that GROUNDED-L is characterised by a pair of forbidden patterns. 
Theorem 1. Consider the two patterns $P_{1}=(\{1,2,3,4\},\{13,24\},\{12,23\})$ and $P_{2}=$ $(\{1,2,3,4\},\{12,14,23\},\{13\})$; see Figure 3. A graph $G=(V, E)$ is a grounded L-graph if and only if it has a vertex ordering that avoids both $P_{1}$ and $P_{2}$. In fact, a linear order $<$ on $V$ avoids the two patterns $P_{1}$ and $P_{2}$ if and only if $G$ has a grounded L-representation which induces the linear order $<$.

Proof. Suppose first that $G$ has a grounded L-representation. Let $\ell_{1}, \ell_{2}, \ldots, \ell_{n}$ be the L-shapes used in the representation, ordered left to right according to the positions of their anchors. Let $h_{i}$ and $v_{i}$ denote, respectively, the horizontal and vertical segment of $\ell_{i}$. Let $x_{i}$ be the vertex represented by $\ell_{i}$. We will show that the vertex ordering $x_{1}<x_{2}<\cdots<x_{n}$ avoids the two patterns $P_{1}$ and $P_{2}$.

Assume that $(G,<)$ contains $P_{1}$, and let $x_{p}<x_{q}<x_{r}<x_{s}$ be the four vertices forming a copy of $P_{1}$. Since $x_{q} x_{s}$ is an edge, the two L-shapes $\ell_{q}$ and $\ell_{s}$ intersect. Let $R$ be the rectangle whose vertices are the anchors of $\ell_{q}$ and $\ell_{s}$, the bend of $\ell_{q}$ and the intersection of $\ell_{q}$ and $\ell_{s}$. Since neither $x_{p}$ nor $x_{r}$ is adjacent to $x_{q}$, we see that $\ell_{p}$ is completely outside of $R$, while $v_{r}$ is inside $R$. It follows that $\ell_{p}$ and $\ell_{r}$ are disjoint, and fail to represent the compulsory edge 13 of $P_{1}$.

Suppose now that $(G,<)$ contains $P_{2}$, and let $x_{p}<x_{q}<x_{r}<x_{s}$ now be the four vertices forming a copy $P_{2}$. Since $x_{p} x_{s}$ is an edge, the segment $h_{p}$ intersects $v_{s}$. Moreover, $v_{q}$ intersects $h_{p}$, while $v_{r}$ does not intersect $h_{p}$, and in particular, $\ell_{q}$ and $\ell_{r}$ fail to represent the compulsory edge 23 of $P_{2}$. We conclude that any grounded L-representation of $G$ induces a vertex order that avoids $P_{1}$ and $P_{2}$.

To prove the converse, assume that $G$ is a graph with a vertex ordering $x_{1}<x_{2}<$ $\cdots<x_{n}$ which avoids both $P_{1}$ and $P_{2}$. We will construct a grounded L-representation $\ell_{1}, \ell_{2}, \ldots, \ell_{n}$ of $G$ inducing the order $<$, with $\ell_{i}$ being the L-shape representing the vertex $x_{i}$.

We fix the anchor of $\ell_{i}$ to be the point $(i, 0)$ on the horizontal axis. Next, we process the vertices left to right, and for a vertex $x_{i}$ we define the representing shape $\ell_{i}$, assuming $\ell_{1}, \ell_{2}, \ldots, \ell_{i-1}$ have already been defined, and assuming further that for any $j<i$ such that $x_{j} x_{i}$ is an edge of $G$, the horizontal segment $h_{j}$ of $\ell_{j}$ reaches to the right of the point $(i, 0)$.

To define $\ell_{i}$, we first describe its vertical segment $v_{i}$. Let $N_{i}^{-}$be the set of vertices $x_{j}$ such that $j<i$ and $x_{j} x_{i} \in E$. If $N_{i}^{-}$is empty, choose the vertical segment $v_{i}$ to be shorter than any of $v_{1}, \ldots, v_{i-1}$. In particular, $v_{i}$ will not intersect any of the L-shapes constructed in previous steps. If $N_{i}^{-}$is nonempty, let $x_{p}$ be a vertex from $N_{i}^{-}$chosen so that $v_{p}$ is as long as possible (and therefore $h_{p}$ is as low as possible). Then define $v_{i}$ to be slightly longer than $v_{p}$, so that $v_{i}$ intersects $h_{p}$ (recall that $h_{p}$ reaches to the right of $(i, 0)$ ) but does not intersect any L-shape whose horizontal segment is below $h_{p}$. This choice of $v_{i}$ guarantees that $v_{i}$ intersects $h_{j}$ for any $x_{j} \in N_{i}^{-}$.

It remains to define the segment $h_{i}$. Let $j$ be the largest index such that $j>i$ and $x_{i} x_{j} \in E$. If no such $j$ exists, set $j=i$. The horizontal segment $h_{i}$ then has length $j-i+\frac{1}{2}$, and in particular, its right endpoint has horizontal coordinate $j+\frac{1}{2}$.

Having defined the L-shapes $\ell_{1}, \ldots, \ell_{n}$ as above, let us verify that their intersection graph is $G$. If $x_{j} x_{i}$ is an edge of $G$ with $j<i$, then the definitions of $v_{i}$ and $h_{j}$ guarantee 

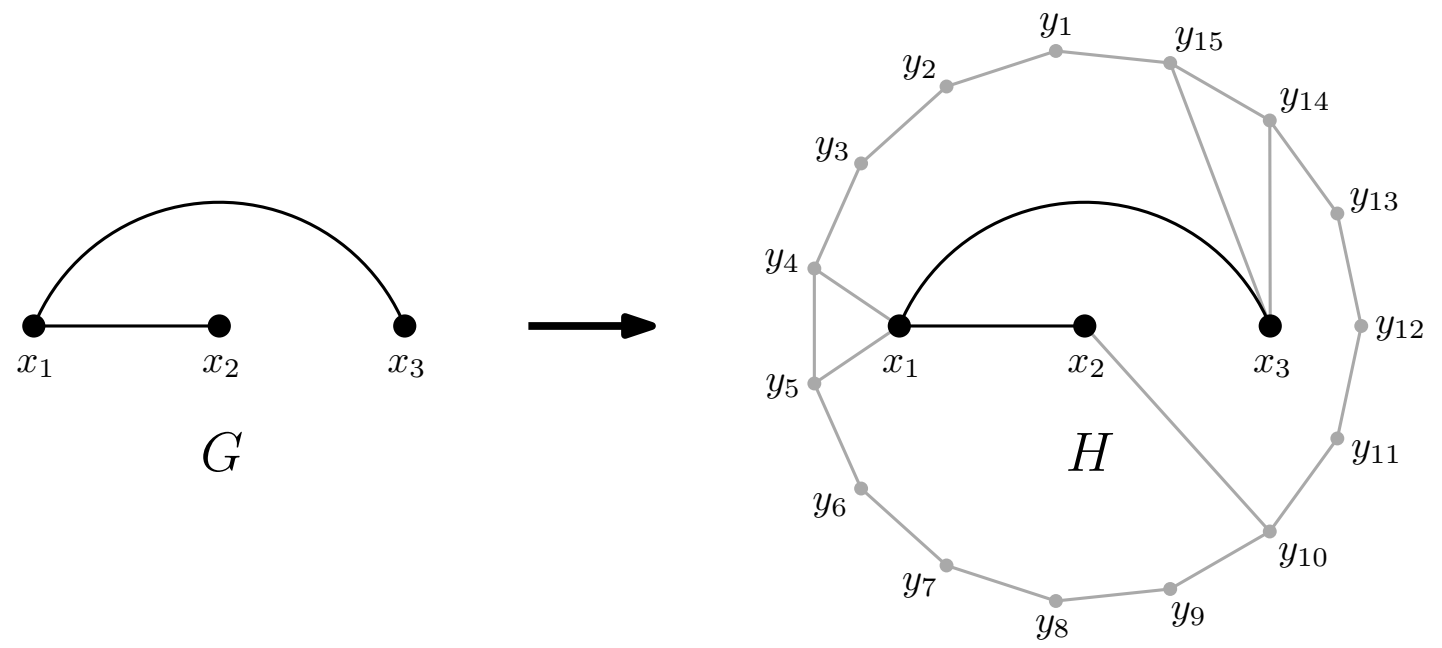

Figure 4: An ordered graph $G$, and an example of its cycle extension $H$.

that $v_{i}$ intersects $h_{j}$, and therefore the two L-shapes $\ell_{j}$ and $\ell_{i}$ intersect.

To prove the converse, suppose for contradiction that for some $j<i$ the two L-shapes $\ell_{j}$ and $\ell_{i}$ intersect while $x_{j} x_{i}$ is not an edge of $G$. Choose such a pair $i, j$ so that $i$ is the smallest possible. There must be an index $k>i$ such that $x_{j} x_{k}$ is an edge of $G$, otherwise $h_{j}$ would be too short to intersect $v_{i}$. Similarly, there must be an index $m<i$ such that $x_{m} x_{i}$ is an edge of $G$, and $v_{m}$ is longer than $v_{j}$, otherwise $v_{i}$ would not be long enough to intersect $h_{j}$.

We now distinguish two cases depending on the relative position of $m$ and $j$. If $m<j$, then $\ell_{m}$ and $\ell_{j}$ are disjoint (recall that $v_{m}$ is longer than $v_{j}$ ) and hence $x_{m} x_{j}$ is not an edge of $G$. It follows that the four vertices $x_{m}<x_{j}<x_{i}<x_{k}$ form the pattern $P_{1}$, a contradiction. Suppose now that $j<m$. It follows that $\ell_{j}$ intersects $\ell_{m}$, and therefore $x_{j} x_{m}$ is an edge of $G$, by the minimality of $i$. Thus, the four vertices $x_{j}<x_{m}<x_{i}<x_{k}$ form the pattern $P_{2}$, which is again a contradiction.

\section{Separations between classes}

Consider again the classes in Figure 1. The inclusions indicated by arrows are either easy to observe or follow from known results that we have pointed out in the introduction. Our goal now is to argue that there are no other inclusions among these classes except those that follow by transitivity from the depicted arrows. In particular, the classes are all distinct.

As our main tool, we will use a lemma which is a slight modification of the 'Cycle Lemma' of Cardinal et al. [4]. The lemma allows us to prescribe the cyclic order of a subset of vertices in an outer-1-string representation of a graph. Let $G=\left(V_{G}, E_{G}\right)$ be a graph on $n$ vertices $x_{1}, x_{2}, \ldots, x_{n}$, and let $<$ be the linear order $x_{1}<x_{2}<\cdots<x_{n}$. The cyclic shift of $<$ is the linear order $<_{s}$ defined as $x_{n}<_{s} x_{1}<_{s} x_{2}<_{s} \cdots<_{s} x_{n-1}$. The reversal of $<$, denoted $<_{r}$, is defined as $x_{n}<_{r} x_{n-1}<_{r} \cdots<_{r} x_{1}$. We say that two linear 

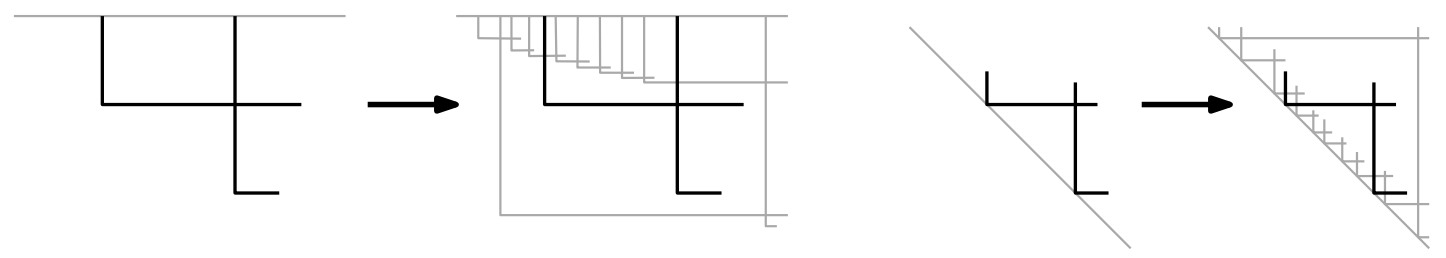

Figure 5: Extending the representation of $G$ into a representation of a cycle extension for grounded L-representations (left) and MPT representations (right).

orders of $V$ are equivalent if one can be obtained from the other by a sequence of cyclic shifts and reversals.

A cycle extension of the ordered graph $(G,<)$ is an (unordered) graph $H=\left(V_{H}, E_{H}\right)$ with the following properties (see Figure 4):

- $V_{H}$ is the disjoint union of the sets $V_{G}=\left\{x_{1}, \ldots, x_{n}\right\}$ and $V_{C}=\left\{y_{1}, \ldots, y_{5 n}\right\}$. The vertices $V_{G}$ induce the graph $G$ (in particular, $E_{G} \subseteq E_{H}$ ), and $V_{C}$ induce a cycle of length $5 n$ with edges $y_{1} y_{2}, y_{2} y_{3}, \ldots, y_{5 n-1} y_{5 n}, y_{5 n} y_{1}$.

- For each vertex $x_{i} \in V_{G}$, either $x_{i}$ is adjacent to $y_{5 i}$ and has no other neighbors in $V_{C}$, or $x_{i}$ is adjacent to $y_{5 i-1}$ and $y_{5 i}$ and has no other neighbors in $V_{C}$.

For the classes of graphs we consider, an intersection representation of a graph $G$ inducing an order < can always be extended into a representation of a cycle extension of $G$, without modifying the curves representing $G$. This is formalised by the next lemma.

Lemma 2. Given a graph class $\mathcal{C} \in\{$ Grounded-L, Grounded- $\{\mathrm{L}\rfloor$,$\} , Grounded-SEG,$ MPT, OUTER-1-STRING $\}$, for every $\mathcal{C}$-representation of a graph $G$ inducing an order < on $V_{G}$ there is a cycle extension $H$ of $(G,<)$ such that a $\mathcal{C}$-representation of $H$ can be constructed by adding the curves representing the vertices of $V_{H} \backslash V_{G}$ into the given $\mathcal{C}$ representation of $G$.

Proof. Suppose we are given a $\mathcal{C}$-representation of $G$. It is easy to see that we can add the curves representing the cycle $V_{C}$ close enough to the grounding line; see Figure 5 . Note that for MPT-representations, each original L-shape may have to be intersected by two consecutive L-shapes from the added cycle. In all the other types of representations, each vertex $x_{i}$ of $G$ will have a unique neighbor $y_{5 i}$ among the $V_{C}$.

Recall that two linear orders are equivalent if one can be obtained from the other by a sequence of cyclic shifts and reversals. The key property of cycle extensions of $(G,<)$ is that they restrict the possible vertex orders of the $G$-part to an order equivalent to $<$, as shown by the next lemma.

Lemma 3. If $(G,<)$ is an ordered graph with a cycle extension $H$, then in every grounded 1 -string representation of $H$, the order of the vertices of $G$ induced by the representation is equivalent to the order $<$. 
Proof. The proof follows the same ideas as the proof of the Cycle Lemma of Cardinal et al. [4, Lemma 4].

Suppose $(G,<)$ is an ordered graph with vertex set $V_{G}=\left\{x_{1}<x_{2}<\cdots<x_{n}\right\}$ and edge set $E_{G}$, and $H$ is its cycle extension, with vertices $V_{H}=V_{G} \cup V_{C}$ as in the definition of cycle extension and $V_{C}=\left\{y_{1}, \ldots, y_{5 n}\right\}$. When working with the indices of the vertices in $V_{C}$, we will assume that arithmetic operations are performed modulo $5 n$, so $5 n+1=1$, etc. Suppose that $H$ has a grounded 1-string representation. We may transform this representation into an outer-1-string representation, while preserving the induced vertex order up to equivalence. Suppose then that an outer-1-string representation of $H$ is given, inside a disk whose boundary is a circle $B$. Let $c_{j}$ be the string representing $y_{j}$, and let $p_{j, j+1}$ be the intersection point of $c_{j}$ and $c_{j+1}$. The subcurve of $c_{j}$ between the two intersection points $p_{j-1, j}$ and $p_{j, j+1}$ is the central part of $c_{j}$, denoted center $(j)$. The part of $c_{j}$ between the anchor and the first point of center $(j)$ is the initial part of $c_{j}$, denoted $\operatorname{start}(j)$. Let $p_{j}$ be the common endpoint of $\operatorname{start}(j)$ and $\operatorname{center}(j)$. Note that $p_{j}$ is equal to $p_{j-1, j}$ or to $p_{j, j+1}$. The sequence of curves center $(1)$, center $(2), \ldots, \operatorname{center}(5 n)$ forms a closed Jordan curve, denoted by $C$. Note that $C$ contains all the points $p_{k, k+1}$ for $k=1, \ldots, 5 n$. Let $R_{C}$ be the interior region of $C$.

Consider now a vertex $x_{i}$, represented by a string $s_{i}$. Note that $s_{i}$ can intersect the curve $C$ only at a point of center(5i) or possibly center $(5 i-1)$. Let $R_{i}$ be the planar region bounded by the union of the following four curves: start $(5 i-3)$, $\operatorname{start}(5 i+2)$, the arc of $C$ between $p_{5 i-3}$ and $p_{5 i+2}$ that contains center $(5 i-1) \cup \operatorname{center}(5 i)$, and the arc of $B$ between the anchors of $c_{5 i-3}$ and $c_{5 i+2}$ that contains the anchors of $c_{5 i-1}$ and $c_{5 i}$.

Note that $s_{i}$ is the only string among the strings representing $V_{G}$ that can intersect the boundary of $R_{i}$. Note also that the string $c_{5 i}$ cannot intersect the boundary of $R_{k}$ for $k \neq i$, and therefore $c_{5 i}$ is contained in $R_{i} \cup R_{C}$. Since $s_{i}$ intersects $c_{5 i}$, and since $s_{i}$ also cannot cross the boundary of $R_{k}$ for $k \neq i$, it follows that $s_{i}$ is also contained in $R_{C} \cup R_{i}$, and in particular, the anchor of $s_{i}$ is in $R_{i} \cap B$. Therefore, the anchors of $s_{1}, \ldots, s_{n}$ appear on $B$ in the order which, up to equivalence, corresponds to the order $<$ on $V_{G}$.

We will now use Lemmas 2 and 3 to construct graphs that have no representation in a given intersection class. Our goal is to show that there are no inclusions missing in Figure 1. The classes Int, Circle, Outerplanar and Per are well studied [2], and simple examples show that there are no inclusions among them other than those depicted in Figure 1.

Catanzaro et al. [5, Observation 6.9] observed that the graph $K_{2,2,2}$ (the octahedron) is a permutation graph not in MPT, and therefore neither PER nor any superclass of PER is contained in MPT. Cardinal et al. [4] showed that GROUNDED-SEG is a proper subclass of OUter-1-STRInG. To complete the hierarchy, we only need the following separations.

Theorem 4. The following properties hold.

(i) The class Grounded- $\{\mathrm{L}\rfloor$,$\} is not a subclass of GROUnDED-L.$

(ii) The class GroundeD-SEG is not a subclass of GROUnDED- $\{\mathrm{L}\lrcorner$,$\} .$

(iii) The class MPT is not a subclass of OUTER-1-STRING. 

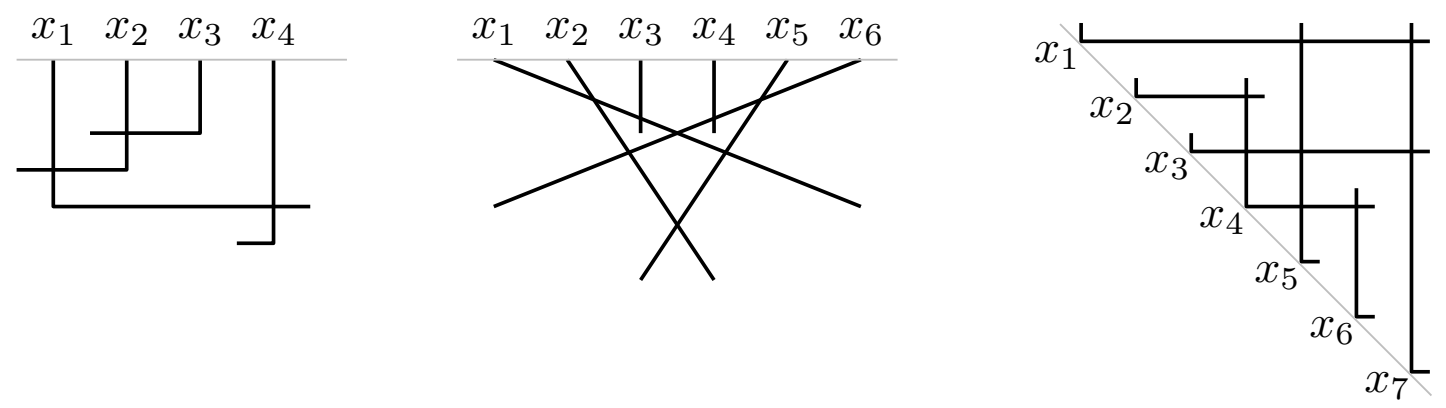

Figure 6: The three intersection representations used to prove Theorem 4 . In each case, a representation cannot be replaced by a representation from a smaller class while preserving the induced vertex order. Left: a GROUNDED- $\{L, J\}$ representation which cannot be replaced by a GROUNDED-L one. Middle: a GROUNDED-SEG representation which cannot be replaced by a GROUNDED- $\{\mathrm{L}\rfloor$,$\} one. Right: an MPT representation which cannot be$ replaced by an OUTER-1-STRING one.

Proof. We first prove part (i) of the theorem. Consider the graph $G=(V, E)$ with $V=\left\{x_{1}, x_{2}, x_{3}, x_{4}\right\}$ and $E=\left\{x_{1} x_{2}, x_{2} x_{3}, x_{1} x_{4}\right\}$. Figure 6 (left) shows a grounded $\left.\{\mathrm{L}\lrcorner,\right\}$ representation of $G$ which induces the order $<$ defined as $x_{1}<x_{2}<x_{3}<x_{4}$ on $V$. Note that there is no grounded L-representation of $G$ that would induce the vertex order $<$, because $(G,<)$ contains the pattern $P_{2}$ of Theorem 1 .

Let $\left(G^{\prime},<^{\prime}\right)$ be the ordered graph obtained by putting $(G,<)$ and the mirror image of $(G,<)$ side by side. Formally, $\left(G^{\prime},<^{\prime}\right)$ has vertex set $V^{\prime}=\left\{x_{1}, x_{2}, x_{3}, x_{4}, y_{1}, y_{2}, y_{3}, y_{4}\right\}$, edge set $E^{\prime}=\left\{x_{1} x_{2}, x_{2} x_{3}, x_{1} x_{4}, y_{1} y_{2}, y_{2} y_{3}, y_{1} y_{4}\right\}$ and vertex order $x_{1}<^{\prime} x_{2}<^{\prime} x_{3}<^{\prime}$ $x_{4}<^{\prime} y_{4}<^{\prime} y_{3}<^{\prime} y_{2}<^{\prime} y_{1}$. Finally, let $\left(G^{\prime \prime},<^{\prime \prime}\right)$ be the ordered graph obtained by placing two disjoint copies of $\left(G^{\prime},<^{\prime}\right)$ side by side. Clearly $G^{\prime \prime}$ has a grounded $\left.\{\mathrm{L}\rfloor,\right\}$ representation which induces the vertex order $<^{\prime \prime}$. However, $G^{\prime \prime}$ has no grounded Lrepresentation inducing a vertex order equivalent with $<^{\prime \prime}$, since in any vertex order equivalent with $<^{\prime \prime}$ there are four consecutive vertices forming a copy of $P_{2}$.

By Lemma 2, the ordered graph $\left(G^{\prime \prime},<^{\prime \prime}\right)$ has a cycle extension $H$ that admits a grounded $\{\mathrm{L}\lrcorner$,$\} -representation. By Lemma 3, any grounded 1-string representation (and$ therefore any grounded L-representation) of $H$ induces on $V^{\prime \prime}$ an order which is equivalent with $<$ ". It follows that $H$ has no grounded L-representation, and therefore GROUNDED$\{\mathrm{L}\lrcorner$,$\} is not a subclass of GROUNDED-L, as claimed.$

For the other two parts of the theorem, the argument is analogous, the main difference is in the choice of the initial ordered graph $(G,<)$. To prove part (ii), consider the graph $G$ on six vertices whose GroundED-SEG representation is in the middle of Figure 6, and let $<$ be the vertex order induced by the depicted representation.

Let us argue that $G$ has no grounded $\{\mathrm{L}\rfloor$,$\} -representation inducing the vertex order <$. For contradiction, suppose that such a representation exists, and let $\ell_{i}$ denote the L-shape or $\lrcorner$-shape representing $x_{i}$ in this representation. Let $h_{i}$ and $v_{i}$ be the horizontal and vertical segments of $\ell_{i}$, respectively. Assume, without loss of generality, that $v_{1}$ is longer than $v_{6}$. Since $\ell_{1}$ and $\ell_{6}$ intersect, $h_{6}$ must intersect $v_{1}$, and $\ell_{6}$ is a $\lrcorner$-shape. Since $\ell_{2}$ 
intersects both $\ell_{1}$ and $\ell_{6}, v_{2}$ must be longer than $v_{6}$, and $v_{2}$ intersects $h_{6}$. But this means that $\ell_{3}$ must intersect either $\ell_{2}$ or $\ell_{6}$ in order to intersect $\ell_{1}$, a contradiction.

Note that the graph $(G,<)$ is isomorphic to its reversal. Consider the ordered graph $\left(G^{\prime},<^{\prime}\right)$ obtained by placing two copies of $(G,<)$ side by side: note that in any vertex order equivalent to $<^{\prime}, G^{\prime}$ contains a copy of $(G,<)$, and therefore there is no grounded $\{\mathrm{L}\lrcorner$,$\} -representation of G^{\prime}$ inducing a vertex order equivalent to $<$. We apply Lemmas 2 and 3 to $\left(G^{\prime},<^{\prime}\right)$ and obtain its cycle extension $H$, which is in GROUNDED-SEG but not in GROUNDED- $\{\mathrm{L}\lrcorner$,$\} .$

To prove part (iii), consider the graph $G$ whose MPT-representation is depicted in the right part of Figure 6, and let $<$ be the vertex order induced by the representation. We claim that there is no grounded 1-string representation of $G$ inducing the order $<$. For contradiction, suppose that such a representation exists, and let $s_{i}$ be the string representing the vertex $x_{i}$. Additionally, let $a_{i}$ denote the anchor of $s_{i}$, and for a pair of intersecting strings $s_{i}, s_{j}$ let $p_{i j}$ denote their intersection.

Assume, without loss of generality, that when we follow $s_{4}$ starting at $a_{4}$, we encounter $p_{24}$ before we encounter $p_{46}$. Let $C$ be the closed Jordan curve obtained as the union of the subcurve of $s_{1}$ between $a_{1}$ and $p_{17}$, the subcurve of $s_{7}$ between $p_{17}$ and $p_{37}$, the subcurve of $s_{3}$ between $p_{37}$ and $a_{3}$, and the segment $a_{1} a_{3}$ of the grounding line. Note that $s_{2}$ is inside $C$ (except $a_{2}$, which lies on $C$ ), and both $a_{4}$ and $s_{6}$ are outside $C$. Therefore, $s_{4}$ must intersect $C$ at least twice: once between $a_{4}$ and $p_{24}$, and once between $p_{24}$ and $p_{46}$. However, $s_{4}$ can only intersect $C$ in the point $p_{34}$, a contradiction.

To complete the proof, we first observe that $G$ has no grounded 1-string representation inducing a vertex order equivalent with $<$, since such a representation could be trivially transformed into a grounded 1-string representation inducing $<$. We apply Lemmas 2 and 3 to $G$, to obtain a graph $H$ which is in MPT but not in Outer-1-STRING.

Note that these results imply that OUTER-STRING is a proper superclass of both MPT and Outer-1-STRING.

We remark that MPT is clearly a subclass of 1-STRING and of OUTER-STRING, but it is not a subclass of OUTER-1-STRING, as we just saw.

\section{Concluding remarks}

We have seen that the vertex orders induced by grounded L-representations can be characterised by a pair of forbidden patterns. Previously, a characterisation by a single forbidden pattern has been found for vertex orders induced by MPT representations $[1,5,15]$. Another related result, due to Pach and Tomon [13], provides a characterisation by a single forbidden pattern for the so-called semi-comparability graphs, which include the complements of the intersection graphs of grounded $y$-monotone curves. It is an open problem whether a characterisation by forbidden patterns can be obtained for other similar grounded intersection classes, such as the class GROUNDED- $\{\mathrm{L}\rfloor$,$\} .$

Another problem concerns the recognition complexity of the graph classes we considered. Recognition of max point-tolerance graphs is mentioned as a prominent open 
problem by Ahmed et al. [1], by Catanzaro et al. [5], as well as by Soto and Thraves Caro [15]. For the classes Grounded-L and Grounded- $\{L\lrcorner$,$\} , recognition is open as$ well. On the other hand, the recognition problem for GROUNDED-SEG is known to be $\exists \mathbb{R}$-complete, as shown by Cardinal et al. [4]. In particular, GROUNDED-SEG cannot be characterised by finitely many forbidden vertex order patterns, unless $\exists \mathbb{R}$ is equal to NP.

The characterisation of GROUNDED-L by forbidden vertex order patterns might conceivably be helpful in designing a polynomial recognition algorithm, but note that even a graph class characterised by a forbidden vertex order pattern may have NP-hard recognition [8], although it is known that recognition is decidable in polynomial time for all classes described by a set of forbidden patterns of order at most three [10].

\section{References}

[1] Abu Reyan Ahmed, Felice De Luca, Sabin Devkota, Alon Efrat, Md Iqbal Hossain, Stephen G. Kobourov, Jixian Li, Sammi Abida Salma, and Eric Welch. L-graphs and monotone L-graphs. arXiv:1703.01544, 2017.

[2] Andreas Brandstädt, Van Bang Le, and Jeremy P. Spinrad. Graph classes: a survey. SIAM Monographs on Discrete Mathematics and Applications. Society for Industrial and Applied Mathematics (SIAM), Philadelphia, PA, 1999.

[3] Sergio Cabello and Miha Jejčič. Refining the hierarchies of classes of geometric intersection graphs. Electron. J. Combin., 24(1):\#P1.33, 19, 2017.

[4] Jean Cardinal, Stefan Felsner, Tillmann Miltzow, Casey Tompkins, and Birgit Vogtenhuber. Intersection graphs of rays and grounded segments. Journal of Graph Algorithms and Applications, 22(2):273-295, 2018.

[5] Daniele Catanzaro, Steven Chaplick, Stefan Felsner, Bjarni V. Halldórsson, Magnús M. Halldórsson, Thomas Hixon, and Juraj Stacho. Max point-tolerance graphs. Discrete Appl. Math., 216(1):84-97, 2017.

[6] Jérémie Chalopin and Daniel Gonçalves. Every planar graph is the intersection graph of segments in the plane: Extended abstract. In Proceedings of the Forty-first Annual ACM Symposium on Theory of Computing, STOC '09, pages 631-638, New York, NY, USA, 2009. ACM.

[7] Peter Damaschke. Forbidden ordered subgraphs. In Topics in combinatorics and graph theory (Oberwolfach, 1990), pages 219-229. Physica, Heidelberg, 1990.

[8] Dwight Duffus, Mark Ginn, and Vojtěch Rödl. On the computational complexity of ordered subgraph recognition. Random Structures Algorithms, 7(3):223-268, 1995.

[9] Daniel Gonçalves, Lucas Isenmann, and Claire Pennarun. Planar graphs as Lintersection or L-contact graphs. In Proceedings of the Twenty-Ninth Annual ACMSIAM Symposium on Discrete Algorithms, SODA '18, pages 172-184, Philadelphia, PA, USA, 2018. Society for Industrial and Applied Mathematics. 
[10] Pavol Hell, Bojan Mohar, and Arash Rafiey. Ordering without forbidden patterns. In Algorithms-ESA 2014, volume 8737 of Lecture Notes in Comput. Sci., pages 554-565. Springer, Heidelberg, 2014.

[11] Sean McGuinness. On bounding the chromatic number of L-graphs. Discrete Math., 154(1-3):179-187, 1996.

[12] Matthias Middendorf and Frank Pfeiffer. The max clique problem in classes of stringgraphs. Discrete Math., 108(1-3):365-372, 1992.

[13] János Pach and István Tomon. On the Chromatic Number of Disjointness Graphs of Curves. In Gill Barequet and Yusu Wang, editors, 35th International Symposium on Computational Geometry (SoCG 2019), volume 129 of Leibniz International Proceedings in Informatics (LIPIcs), pages 54:1-54:17, Dagstuhl, Germany, 2019. Schloss Dagstuhl-Leibniz-Zentrum fuer Informatik.

[14] Alexandre Rok and Bartosz Walczak. Outerstring graphs are $\chi$-bounded. In Computational geometry (SoCG'14), pages 136-143. ACM, New York, 2014.

[15] Mauricio Soto and Christopher Thraves Caro. p-box: a new graph model. Discrete Math. Theor. Comput. Sci., 17(1):169-186, 2015.

[16] Walter Wessel and Reinhard Pöschel. On circle graphs. In Graphs, hypergraphs and applications (Eyba, 1984), volume 73 of Teubner-Texte Math., pages 207-210. Teubner, Leipzig, 1985. 